\title{
IMPACT OF THE SOCIOECONOMIC STATUS ON SURVIVAL AFTER ACUTE ISCHEMIC STROKE: A POPULATION-BASED STUDY IN A REGION WITH UNIVERSAL HEALTHCARE COVERAGE
}

Rosa Maria Vivanco-Hidalgo, Aida Ribera, Mercè Salvat, Guillem Gallofré, Lluís Martínez, Miquel Gallofré, Sònia Abilleira Stroke Programme/ Agency for Health Quality and Assessment of Catalonia

Background: Differences in stroke mortality by socioeconomic status (SES) exist but are heterogeneous in high-income countries. Objective: to assess the impact of SES on 30-day and longer-term mortality in acute ischemic stroke (AIS) patients living in a region with universal healthcare coverage.

Methods: We used healthcare administrative data to identify AIS hospitalizations (index cases, ICD-9: 433.x1, 434.x1, 436) occurred among Catalonia's residents between Jan15-Dec16. Data included age, gender, reperfusion therapies (99.10, 39.74), and the basic healthcare area of residence (BHCAR). Patients vital status was followed up until Sep17 through linkage to the Central Registry of Insured Persons. The SES index consists of 9 components and reflects socioeconomic differences between BHCAR. Adjusted logistic and Cox regression models using the Huber-White cluster sandwich estimator of variance were used to analyze the effect of SES (per quartiles) on survival.

Results: We analyzed 16,669 AIS patients. Those living in BHCAR with high SES were older (mean 76 years, SD 13 vs 73 years, SD 13; $p$-value: <0.001), and received higher treatment rates than those living in BHCAR with lower SES $(17 \%$ vs 15.6\%; p-value: 0.08) (Table 1). During follow-up 5,174 died (31\%). Patients living in low SES areas had higher adjusted 30day mortality rates compared to those living in higher SES areas (OR: 1.22 [1.08-1.38], p<0.01). No differences between SES quartiles were observed in >30-day mortality (HR: 1.01 [0.911.13]; $\mathrm{p:}$ : 0.81). However, differences where observed when analyzing total mortality (HR: 1.09 [1.004-1.18]; $p<0,01)$ (Figure 1).
Conclusion: In our study population, SES plaid a role in 30-day mortality and total mortality. More studies are needed to explain the mechanism of this associations.

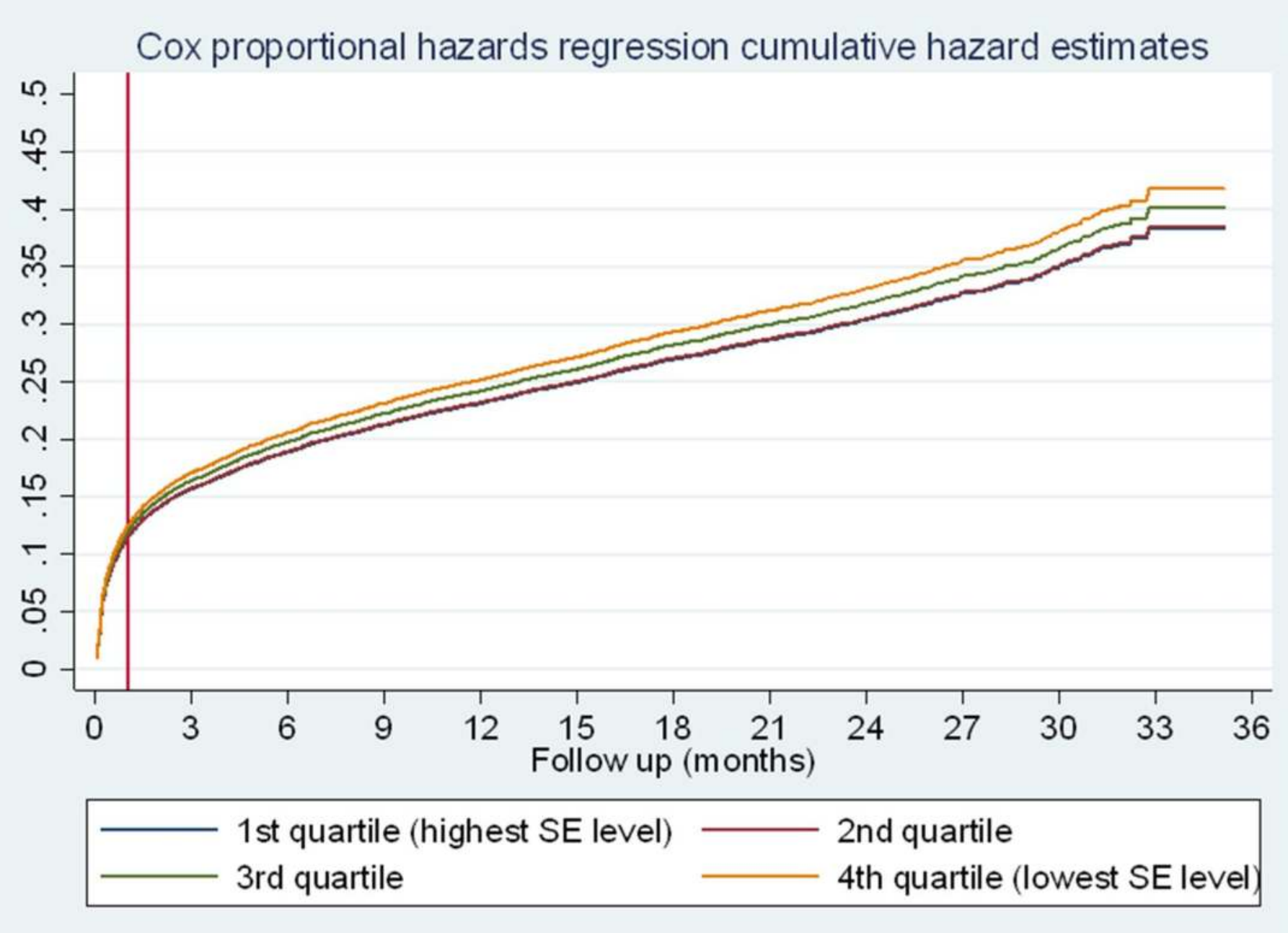

Figure 1. Adjusted total mortality rates per socioeconomic status

\begin{tabular}{|c|c|c|c|c|c|c|}
\hline & $\begin{array}{c}\text { Total } \\
N=16,669\end{array}$ & $\begin{array}{c}1^{\text {st }} \text { quartile } \\
\text { (highest) } \\
\mathrm{N}=4588\end{array}$ & $\begin{array}{c}2^{\text {nd }} \text { quartile } \\
N=4090\end{array}$ & $\begin{array}{c}3^{\text {rd }} \text { quartile } \\
N=3839\end{array}$ & $\begin{array}{c}4^{\text {th }} \text { quartile } \\
\text { (lowest) } \\
\mathrm{N}=4152\end{array}$ & P for trend \\
\hline Women, N (\%) & 7757 (46.5) & 2266 (49.4) & $1872(45.8)$ & 1709 (44.5) & $1910(46)$ & 0.001 \\
\hline Age, mean (sd) & $75(13.4)$ & $76.3(13)$ & $75.2(13.1)$ & $74.4(13.3)$ & $73.7(13.4)$ & $<0.001$ \\
\hline SE level, mean (sd) & $44.9(16.4)$ & $25.2(9.8)$ & $41.7(2.7)$ & $50(2.3)$ & $65(9.8)$ & $<0.001$ \\
\hline Treated, N (\%) & $2688(16.1)$ & 777 (16.9) & $664(16.2)$ & $598(15.6)$ & $649(15.6)$ & 0.079 \\
\hline Thrombolysis & $1691(63.3)$ & $486(62.9)$ & $431(65.6)$ & $379(63.9)$ & 395 (61) & \\
\hline Rescue thrombectomy & $517(19.4)$ & $140(18.1)$ & $112(17.1)$ & $108(18.2)$ & $148(22.8)$ & \\
\hline Direct thrombectomy & $463(17.3)$ & 147 (19) & $114(17.4)$ & $106(17.9)$ & $105(16.2)$ & \\
\hline 30d mortality, $\mathrm{N}(\%)$ & 2488 (14.9) & 686 (15) & 594 (14.5) & 591 (15.4) & 617 (14.9) & 0.002 \\
\hline
\end{tabular}

Table 1. Patients characteristics per socioeconomic status 